\title{
Diet at age 10 and 13 years in children identified as picky eaters at age 3 years in a longitudinal birth cohort study
}

\author{
C.M. Taylor and P.M. Emmett \\ Centre for Child and Adolescent Health, School of Social and Community Medicine, University of Bristol, UK
}

\section{This abstract was presented as the Public Health Nutrition Theme Highlight}

Picky eating is characterised by an unwillingness to eat familiar foods or to try new foods, as well as strong food preferences. ${ }^{(1,2)}$ The peak age for prevalence is during preschool years. ${ }^{(1)}$ While picky eating may result in alterations in the intake of energy ${ }^{(3)}$ and some micronutrients ${ }^{(4,5)}$ at around this age, there are very few studies of longer term dietary outcomes associated with picky eating identified in preschool years. The aim of the present study was to characterise the diets of children aged $3 \cdot 5,10$ and 13 years who had been identified as picky eaters at age 3 years and compare them with the diets of non-picky children. Differences in food group and nutrient intakes between the picky eating groups were compared across the three ages.

Picky eaters were identified in the Avon Longitudinal Study of Parents and Children (ALSPAC) database from a questionnaire item (Does your child have definite likes and dislikes as far as food is concerned? No/Yes, quite choosy/Yes, very choosy) completed by the caregiver when the child was 3 years old. Dietary intake was assessed at age 3.5 years with 3-day food records completed by the caregiver. This was repeated at age 10 and 13 years with completion by the child with parental help. The food records were coded and analysed as described by Emmett et al. ${ }^{(6)}$

As previously reported, at age 3.5 years, children who were picky eaters had lower intakes of meat, fruit and vegetables than those who were not picky eaters (Table 1). This was reflected in differences in protein, carotene, selenium, iron, zinc and dietary fibre intake between the two groups. ${ }^{(4)}$ There were similar differences to those at age 3.5 years in food group intakes at 10 and 13 years of age, in particular lower intakes of meat and vegetables in picky children than in children who were not picky. At 13 years old there were differences in vegetable (23\% lower in picky children), fruit (14\% lower) and total meat (8\% lower) intakes (Table 1$)$.

Table 1. Food group intakes (g/day) assessed by food records at 3.5, 10 and 13 years by picky eating score assessed by questionnaire at 3 years

\begin{tabular}{|c|c|c|c|c|c|c|}
\hline & \multicolumn{2}{|c|}{ Age 3.5 years $^{\mathrm{a}}$} & \multicolumn{2}{|c|}{ Age 10 years } & \multicolumn{2}{|c|}{ Age 13 years } \\
\hline & Not picky eater & Picky eater & Not picky eater & Picky eater & Not picky eater & Picky eater \\
\hline $\mathrm{n}$ & 364 & 131 & 2341 & 804 & 1981 & 693 \\
\hline Total meat (g/day) & $61(57,65)$ & $50(43,57)^{*}$ & $107(104,110)$ & $91(87,95)^{*}$ & $125(122,129)$ & $115(109,122)^{*}$ \\
\hline Meat, carcass $(\mathrm{g} / \text { day })^{\mathrm{b}}$ & $38(34,43)$ & $26(20,32)^{*}$ & $60(58,62)$ & $44(41,48)^{*}$ & $76(73,79)$ & $69(64,75)$ \\
\hline Processed meat $(\mathrm{g} / \text { day })^{\mathrm{c}}$ & $23(20,25)$ & $24(20,28)$ & $47(45,49)$ & $47(44,50)$ & $49(47,52)$ & $46(43,50)$ \\
\hline Total fish (g/day) & $16(14,18)$ & $9(7,12)^{*}$ & $14(13,15)$ & $11(10,13)^{*}$ & $16(15,17)$ & $14(12,16)$ \\
\hline Total vegetables (g/day) & $52(48,57)$ & $25(19,31)^{*}$ & $75(73,78)$ & $50(47,54)^{*}$ & $91(88,94)$ & $70(65,75)^{*}$ \\
\hline Total fruit (g/day) & $72(65,78)$ & $46(36,56)^{*}$ & $72(69,75)$ & $63(58,68)^{*}$ & $80(76,84)$ & $69(63,76)^{*}$ \\
\hline Total milk (g/day) $^{\mathrm{d}}$ & $325(305,344)$ & $390(342,437)^{*}$ & $214(207,221)$ & $226(212,240)$ & $224(214,234)$ & $228(211,244)$ \\
\hline
\end{tabular}

Values are mean $(95 \%$ confidence interval).

${ }^{a}$ Data at age 3.5 years previously reported. ${ }^{(4)}$

${ }^{\mathrm{b}}$ Foods included carcass meat from lamb, pork, beef, poultry, liver and kidney.

${ }^{\mathrm{c}}$ Foods included sausages, ham, bacon, burgers, meat pies, breaded poultry, salami, etc.

${ }^{\mathrm{d}}$ Milks included whole, semi-skimmed, skimmed cows' milk, other animal milks, soya milk, human milk, formula and cream.

Value at same age significantly different from that of children who were not picky eaters, $* p<0.05$

At age 10 years there was evidence of similar differences in nutrient intakes to those identified at age $3 \cdot 5$ years, but these differences were generally less evident at age 13 years except for higher free sugars and lower zinc and dietary fibre intakes.

The differences in food and food group intakes, and in nutrient intakes evident at 3.5 years between children who were picky eaters or not at age 3 years showed evidence of persisting into adolescence particularly for vegetable, fruit and meat intakes. More effective strategies focused on helping parents to widen the food choices of their children at an early age need to be developed. These strategies should focus particularly on vegetable and fruit intake.

This work was funded by Nestlé Nutrition.

1. Taylor CM, Wernimont SM, Northstone K et al. (2015) Appetite 95, 349-359.

2. Dovey T, Staples P, Gibson E et al. (2008) Appetite 50, 181-193.

3. van der Horst K, Deming DM, Lesniauskas R et al. (2016) Appetite 103, 286-293.

4. Taylor CM, Northstone K, Wernimont SM et al. (2016) Am J Clin Nutr 104, 1647-1656.

5. Galloway AT, Fiorito L, Lee Y et al. (2005) J Am Diet Assoc 105, 541-548.

6. Emmett P, Rogers I, Symes C et al. (2002) Public Health Nutr 5, 55-64. 